\title{
Cost-effectiveness of paclitaxel, doxorubicin, cyclophosphamide and trastuzumab versus docetaxel, cisplatin and trastuzumab in new adjuvant therapy of breast cancer in china
}

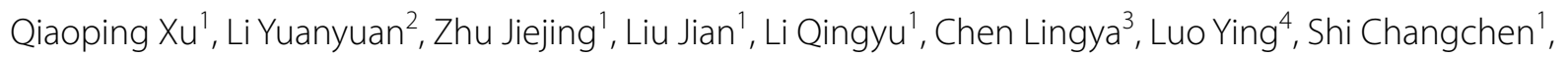
Li Yangling ${ }^{1}$ and Yan Wei ${ }^{1 *}$

\begin{abstract}
Background: Breast cancer is the most common cancer among women in China. Amplification of the Human epidermal growth factor receptor type 2 (HER2) gene is present and overexpressed in 18-20\% of breast cancers and historically has been associated with inferior disease-related outcomes. There has been increasing interest in de-escalation of therapy for low-risk disease. This study analyzes the cost-effectiveness of Doxorubicin/ Cyclophosphamide/ Paclitaxel/ Trastuzumab (AC-TH) and Docetaxel/Carboplatin/Trastuzumab(TCH) from payer perspective over a 5 year time horizon.

Methods: A half-cycle corrected Markov model was built to simulate the process of breast cancer events and death occurred in both AC-TH and TCH armed patients. Cost data came from studies based on a Chinese hospital. One-way sensitivity analyses as well as second-order Monte Carlo and probabilistic sensitivity analyses were performed.The transition probabilities and utilities were extracted from published literature, and deterministic sensitivity analyses were conducted.
\end{abstract}

Results: We identified 41 breast cancer patients at Hangzhou First People's Hospital, among whom 15 (60\%) had a partial response for AC-TH treatment and $13(81.25 \%)$ had a partial response for TCH treatment.No cardiac toxicity was observed. Hematologic grade 3 or 4 toxicities were observed in 1 of 28 patients. Nonhematologic grade 3 or 4 toxicities with a reverse pattern were observed in 6 of 29 patients. The mean QALY gain per patient compared with TCH was 0.25 with AC-TH, while the incremental costs were $\$$ US13,142. The incremental cost-effectiveness ratio (ICER) of AC-TH versus TCH was \$US 52,565 per QALY gained.

Conclusions: This study concluded that TCH neoadjuvant chemotherapy was feasible and active in HER2-overexpressing breast cancer patients in terms of the pathological complete response, complete response, and partial response rates and manageable toxicities.

Keywords: Breast cancer, Neoadjuvant chemotherapy, Pharmaceutical economics, Markov model, Cost-effectiveness analysis

*Correspondence: xqp1984@126.com

1 Department of Pharmacy, Hangzhou First People's Hospital, School of Medicine, Zhejiang University, Hangzhou 310006, China

Full list of author information is available at the end of the article

\section{Background}

Breast cancer is one of the most common malignant tumors in women, and its incidence continues to rise

c) The Author(s) 2021. This article is licensed under a Creative Commons Attribution 4.0 International License, which permits use, sharing, adaptation, distribution and reproduction in any medium or format, as long as you give appropriate credit to the original author(s) and the source, provide a link to the Creative Commons licence, and indicate if changes were made. The images or other third party material in this article are included in the article's Creative Commons licence, unless indicated otherwise in a credit line to the material. If material is not included in the article's Creative Commons licence and your intended use is not permitted by statutory regulation or exceeds the permitted use, you will need to obtain permission directly from the copyright holder. To view a copy of this licence, visit http://creativecommons.org/licenses/by/4.0/. The Creative Commons Public Domain Dedication waiver (http://creativecommons.org/publicdomain/zero/1.0/) applies to the data made available in this article, unless otherwise stated in a credit line to the data. 
globally. In the worldwide, about 1.2 million women have breast cancer every year, accounting for $18 \%$ of all female tumors. The incidence of breast cancer in China also shows an increasing trend year by year [1]. Breast cancer remains the most prevalent invasive cancer among women.China is no exception:

figures showed that the five most commonly diagnosed cancers among women were breast,lung and bronchus,stomach,colorectal, and esophagus cancer.

Trastuzumab, a humanized monoclonal antibody that targets the extracellular domain of the HER-2 protein, was found to improve survival in the metastatic disease setting when used in combination with chemotherapy [2]. Alone and in combination with chemotherapy,trastuzumab has been shown to be safety and active in advanced HER2-positive disease. Then, in a large, randomized study, the addition of trastuzumab to chemotherapy improve the rates of objective response, response duration, and time to disease progression, as well as a $30 \%$ improvement in the rate of overall survival among patients with firstline metastatic disease [2]. A significant side effect was cardiac dysfunction, including congestive heart failure, especially when trastuzumab was used in combination with anthracycline-based regimens [2].

At a median follow up of 36 months, the BCIRG 006 trial [3] had recently demonstrated that every 3 weekly Doxorubicin/Cyclophosphamide (AC) followed by the Paclitaxel (T) group had nominally fewer DFS events and OS events than the Docetaxel/Carboplatin/Trastuzumab $(\mathrm{TCH})$ group, but the differences were not statistically significant and the toxicity profiles differed especially regarding cardiac events. In the BCIRG006 trial (trastuzumab plus chemotherapy in Early Breast Cancer [EBC]), the incidence of congestive heart failure was 2.0 and $0.4 \%$ in the anthracycline and nonanthracycline arms, respectively [3]. It was also anticipated that $\mathrm{TCH}$ might have less cardiac toxicity than anthracycline-based regimens [2].

Randomized Phase III Study of TCH in patients with advanced disease showed that this regimen resulted in the longest period of objective response rate (ORR) and progression free survival (PFS) in women with HER2-overexpressing metastatic breast cancer(MBC), with rare cardiac dysfunction [4].

In prospective studies, Russell et al. [5] summarized that the incidence of symptomatic heart failure events was $2.0 \%$ in patients treated with $\mathrm{AC}-\mathrm{TH}$,and the majority of these patients recovered with appropriate treatment. In the same year, another paper also reported that the incidence of heart failure (CHF) in patients treated with $\mathrm{AC}-\mathrm{TH}[6]$ was $2 \%$ to $4 \%$. The risk benefit ratio favored the nonanthracycline $\mathrm{TCH}$ regimen over
AC-T plus trastuzumab, given its similar efficacy, fewer acute toxic effects, and lower risks of cardiotoxicity, and the incidence of the heart failure (CHF) was $0.4 \%[7$, 8]. Therefore, docetaxel, carboplatin, and trastuzumab (TCaH) is an option for HER2-positive patients who can not use anthracycline. However, carboplatin toxicity could be a concern in certain patients. The primary determinant of carboplatin clearance was the glomerular filtration rate (GFR), which was often decreased in elderly patients and may increase the risk of toxicity [9].

To determine the worth and safety of omitting an anthracycline, a randomized trial of dose-dense AC followed by TH compared with DCT (TCH) could be considered. Therefore, we reviewed our experience concerning the safety and tolerability of standard trastuzumab-containing chemotherapy regimens $(\mathrm{AC}-\mathrm{TH}$ and $\mathrm{TCH}$ ) in early-stage, resectable, HER2-positive breast cancer patients. The aim of this paper was to evaluate the cost effectiveness of adjuvant AC-TH compared with $\mathrm{TCH}$ in the hospital setting for women with early (regional) breast cancer to help guide funding decisions for incurring breast cancer.

\section{Methods \\ Statement}

According to the ministry of health "measures on ethical review of biomedical research involving human beings (trial 2007)" 、WMA 《Declaration of Helsinki》 and the ethical principles of CIOMS 《the international moral guide to human biological research $\rangle$, Subject to review by the ethics committee, Agree to carry out this study according to the research scheme under review.

All methods were carried out in accordance with relevant guidelines and regulations. All experimental protocols were approved by Ethics committee of hangzhou first people's hospital. Informed consent was obtained from all subjects. The protocol of the trial was registered prior to initiation. (The trial registration number: [2014] Scientific Research Medical Ethics No. (028) -01).

\section{Design and structure of the model}

We constructed a Markov model using TreeAge Pro Suite 2011 (TreeAge Software Inc., Williamstown, MA, USA) to analyze the cost-effectiveness of a novel adjuvant $\mathrm{AC}-\mathrm{TH}$ to the $\mathrm{TCH}$ treatment for patients with HER-2-positive breast cancer. In the model, presented in Fig. 1, the patients move between the following four health states: remission, stabilization, relapse and death. Movements between health states were based on transition probabilities that were calculated from clinical trials with a one-year cycle length and adjusted to half cycle in each health state process. The time horizon was five years 
based on the clinical literature.I state that the probabilities came from literature.

Patients accumulated both the costs and qualityadjusted life-years (QALYs) for each period spent in a certain health state. We discounted both the costs and QALYs at a 5\% rate. Since the time value of money is different, discount rate has a certain influence on the calculation of cost. In the baseline analysis, $5 \%$ discount rate is used to calculate the cost, and in the sensitivity analysis, $0 \%-5 \%$ discount rate is used to calculate the incremental cost utility ratio, so as to investigate the stability of the results.The results were presented in incremental cost-effectiveness ratios (ICERs) in terms of the cost per QALY gained. To perform probabilistic sensitivity analysis (PSA), we simulated the model using second-order Monte Carlo analysis (1000 simulations) and constructed confidence intervals with bootstrap replications (1000 replications).

\section{Patients and treatment plans}

We identified 41 patients treated with neoadjuvant trastuzumab using our institutional database from January 2014 to November 2016. All the patients were confirmed to have HER2-positive breast cancer by either immunohistochemistry or fluorescence in situ hybridization. Chemotherapy regimens were selected according to physician preference. Twenty-five patients received AC-TH, and sixteen patients received TCH.

Patients in the AC-TH group received doxorubicin $\left(60 \mathrm{mg} / \mathrm{m}^{2}\right)$ and cyclophosphamide $\left(600 \mathrm{mg} / \mathrm{m}^{2}\right)$ every 2 or 3 weeks for four cycles, followed by weekly paclitaxel $\left(80 \mathrm{mg} / \mathrm{m}^{2}\right)$ for 12 weeks. Trastuzumab was administered with the first dose of paclitaxel at a dose of $4 \mathrm{mg} / \mathrm{kg}$ body weight, followed by $2 \mathrm{mg} / \mathrm{kg}$ body weight weekly during chemotherapy. In the $\mathrm{TCH}$ regimen, patients received $75 \mathrm{mg} / \mathrm{m}^{2}$ of docetaxel and $6 \mathrm{mg}$ per $\mathrm{ml} / \mathrm{min}$ of carboplatin (area under the curve) once every three weeks for six cycles. Trastuzumab was given concurrently with this treatment and consisted of a $4 \mathrm{mg} / \mathrm{kg}$ loading dose followed by $2 \mathrm{mg} / \mathrm{kg}$ weekly for 12 weeks. Thereafter, the dosage became $6 \mathrm{mg} / \mathrm{kg}$ once every three weeks until a one-year course was completed.

Patients with a high risk of febrile neutropenia treated with standard AC-TH (every 3 weeks)received granulocyte-colony stimulating factor (G-CSF) [23]. Patients who developed asymptomatic cardiotoxicity(decrease in left ventricle ejection fraction) were treated with a course of angiotensin-converting enzyme (ACE) inhibitors and another echo. Patients who experienced symptomatic cardiotoxicity (congestive heart failure) were treated with ACE inhibitors, beta blockers and another echo [24] such as dexrazoxane.

\section{Model inputs for transition probabilities}

Transition probabilities, sourced from clinical trials, are presented in Table 1. All the hazard ratios and survival rates were converted into transition probabilities for oneyear time periods.

We calibrated the transition probabilities from our actual overall survival (OS) data and median time to progression using the equation developed by the log-logistic models [10-12] and inputed into the Markov model. The modeled graphical OS data were extracted using a validated graphical digitizer (Web Plot Digitizer version 3.4; Ankit Rohatgi, Austin, TX, USA). The difference between actual OS data and model data derived from our Markov states was compared using chi-squared test. The results represented the internal validation of our model (See Table 2). Health state utilities referred to those used in previously published cost-effectiveness analyses of trastuzumab [13]. We assumed these hazard ratios for diseasefree survival corresponded to a five-year period, similar to the BCIRG 006 data for trastuzumab.

\section{Model inputs for effectiveness}

The utilities that used in the model are presented in Table 1 . Values for the utility associated with health states were taken from a study by Ward et al. [13]. These utilities were chosen because of the similarities in setting.

\section{Model inputs for costs}

All the drug costs came from the Hangzhou First People's Hospital, China. The costs of the treatments were calculated considering the patient body weight and body surface area. We assigned costs attributable to chemotherapy to the first cycle in the Markov model.

\section{Simulations and uncertainty}

By calculating a weighted average using the heterogeneity distribution, incremental QALYs and costs were obtained for all expected breast cancer patients diagnosed in 2015. The analyses were performed using TreeAge Pro 2011.

Monte Carlo simulation was used to address parameter uncertainty, with 1000 draws from input parameters based on the following distributions: log-Normal distribution for the HR for death; beta distributions for the proportions experiencing a toxicity and disability weights; gamma distributions for costs (see Table 2). We reran the models (expected values only; no uncertainty about input parameters) for a range of scenarios to assess the impact of the following structural assumptions:

- discount rate 0 or $5 \%$ per annum.

- exclusion of prevalent life-years with QALYs. 


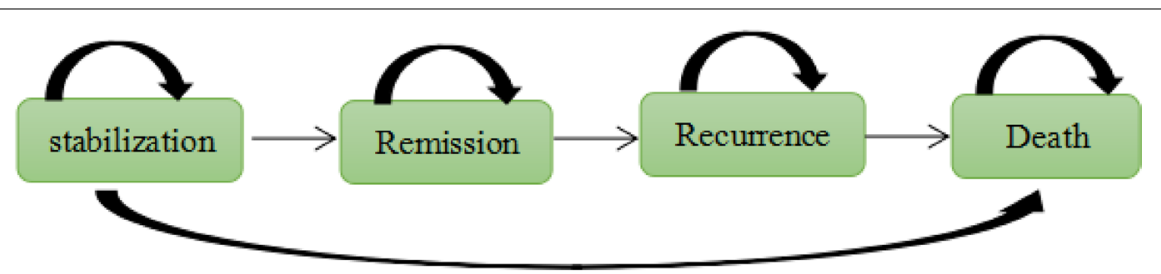

a Markov Model

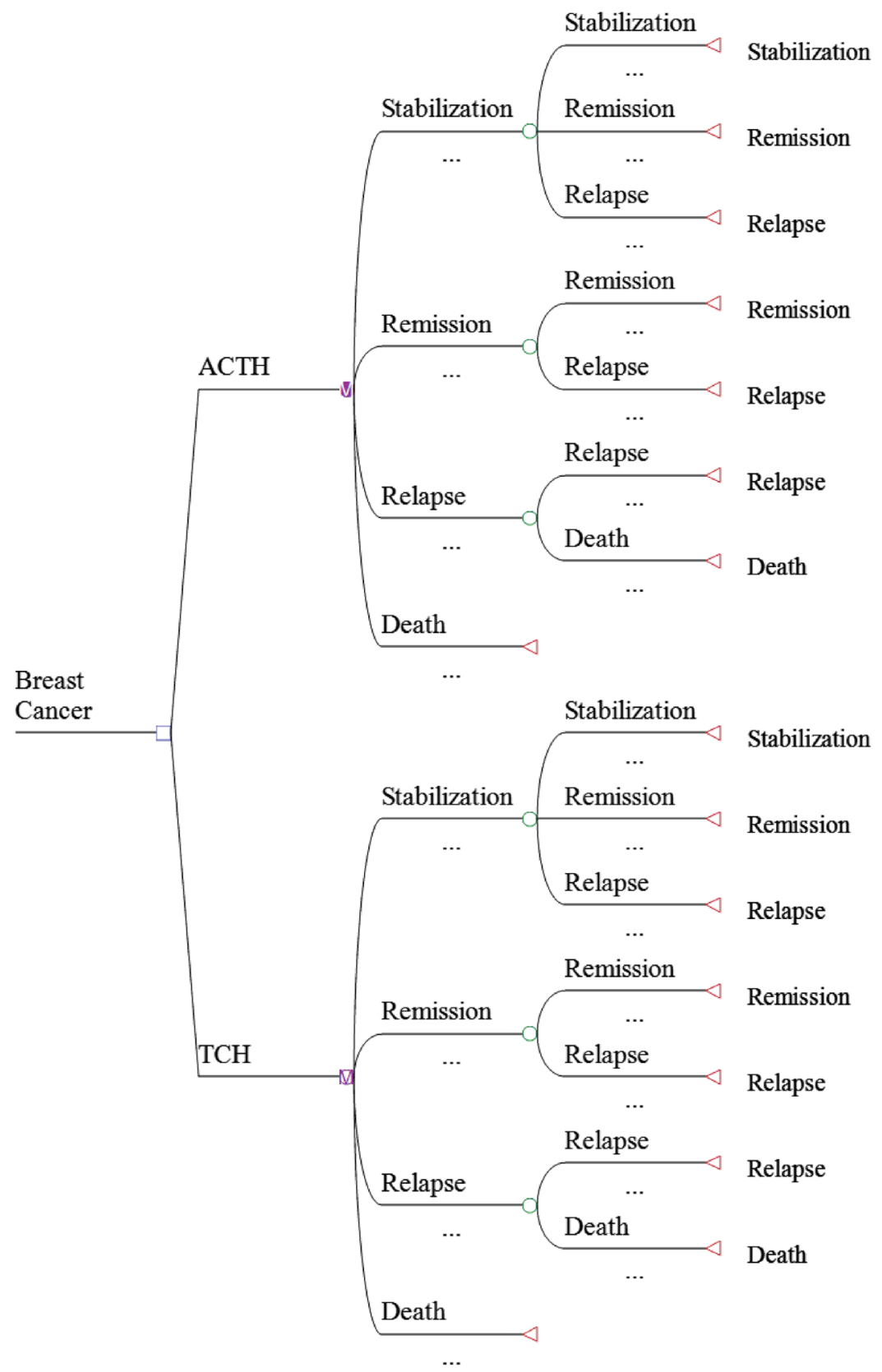

b The Decision Tree.

Fig 1. Schematic of the Decision Tree and Markov Model. ACTH Doxorubicin/Cyclophosphamide/Paclitaxel/Trastuzumab, TCH Docetaxel/ Carboplatin/Trastuzumab) 
Table 1 Key parameters for the model of adjuvant treatment of regional breast cancer with ACTH and TCH

\begin{tabular}{|c|c|c|c|c|c|}
\hline Variable & Formula & Best estimate & SA range & Distribution & Source \\
\hline \multicolumn{6}{|l|}{ Probability } \\
\hline $\begin{array}{l}-\mathrm{ACTH} \\
\mathrm{RR}\end{array}$ & & 0.837 & & & [14-18] \\
\hline $\begin{array}{l}\text { OS } \\
\text { TTP }\end{array}$ & & $\begin{array}{l}44.3 \\
7.2\end{array}$ & & & \\
\hline DOR & & 5.6 & & & \\
\hline Stable $\rightarrow$ Stable (ACss) & 1-ACsp-ACsr & 0.401 & $0.3208-0.4812$ & Beta & \\
\hline Stable $\rightarrow$ Remission (ACsr) & $1-\exp (-\mathrm{RR} / 3)$ & 0.243 & $0.1944-0.2916$ & Beta & \\
\hline Stable $\rightarrow$ Relapse(ACsp) & $\mathrm{ACrp}^{*} 4$ & 0.356 & $0.2848-0.4272$ & Beta & \\
\hline Remission $\rightarrow$ Remission (ACrr) & 1-ACrp & 0.911 & $0.7288-1.0000$ & Beta & \\
\hline Remission $\rightarrow$ Relapse (ACrp) & $\exp (-0.75 * \ln (2) /(5.6))$ & 0.089 & $0.0712-0.1068$ & Beta & \\
\hline Relapse $\rightarrow$ Relapse (ACpp) & 1-ACpd & 0.986 & $0.7888-1.0000$ & Beta & \\
\hline Relapse $\rightarrow$ Death (ACpd) & $\exp \left(-0.75^{*} \ln (2) /(44.3-7.2)\right)$ & 0.014 & $0.0112-0.0168$ & Beta & \\
\hline $\begin{array}{l}-\mathrm{TCH} \\
\mathrm{RR}\end{array}$ & & 0.704 & & & [19-23] \\
\hline $\begin{array}{l}\text { OS } \\
\text { TTP }\end{array}$ & & $\begin{array}{l}35 \\
10.35\end{array}$ & & & \\
\hline DOR & & 4 & & & \\
\hline Stable $\rightarrow$ Stable (TCss) & 1-TCsp-TCsr & 0.311 & $0.2488-0.3732$ & Beta & \\
\hline Stable $\rightarrow$ Remission(TCsr) & 1-exp $(-\mathrm{RR} / 3)$ & 0.209 & $0.1672-0.2508$ & Beta & \\
\hline Stable $\rightarrow$ Relapse(TCsp) & TCrp*4 & 0.48 & $0.384-0.576$ & Beta & \\
\hline Remission $\rightarrow$ Remission (TCrr) & 1-TCrp & 0.88 & $0.704-1.0000$ & Beta & \\
\hline Remission $\rightarrow$ Relapse (TCrp) & $\left.\exp \left(-0.75^{*} \ln (2) / 4.2\right)\right)$ & 0.12 & $0.096-0.144$ & Beta & \\
\hline Relapse $\rightarrow$ Relapse(TCpp) & $1-\mathrm{TCpd}$ & 0.979 & $0.7832-1.0000$ & Beta & \\
\hline Relapse $\rightarrow$ Death(TCpd) & $\exp \left(-0.75^{*} \ln (2) /(35-10.35)\right)$ & 0.021 & $0.0168-0.0252$ & Beta & \\
\hline Discount rate for costs and QALYs & & $5 \%$ per year & & & [24] \\
\hline Health state utilities & & & & & [13] \\
\hline No recurrence (chemotherapeutic period) & & 0.74 & $0.592-0.888$ & Beta & \\
\hline No recurrence (after chemotherapy) & & 0.94 & $0.752-1.000$ & Beta & \\
\hline local recurrence (in the first year) & & 0.74 & $0.592-0.888$ & Beta & \\
\hline remission & & 0.85 & $0.68-1.000$ & Beta & \\
\hline Relapse & & 0.5 & $0.4-0.6$ & Beta & \\
\hline
\end{tabular}

OS overall survival, DOR duration of response, TTP time to progression

Table 2 Cost and effect of ACTH and TCH within five years

\begin{tabular}{|c|c|c|c|c|}
\hline Item & Status & АCTH & $\mathrm{TCH}$ & Deviation \\
\hline \multirow[t]{3}{*}{ Effect } & $\begin{array}{l}\text { Disease Free Sur- } \\
\text { vival/\% }\end{array}$ & $38 \%$ & $46 \%$ & -8 \\
\hline & Death $/ \%$ & $2.6 \%$ & $1.4 \%$ & 1.2 \\
\hline & QALY/Year & 3.4 & 3.65 & -0.25 \\
\hline $\begin{array}{l}\text { Intervention } \\
\operatorname{costs}^{\mathrm{a}}(\$)\end{array}$ & & 3112 & 1352 & \\
\hline $\begin{array}{l}\text { Health system } \\
\text { costs }(\$)\end{array}$ & & 56,425 & 45,043 & \\
\hline $\operatorname{Cost}(\$)$ & & 59,537 & 46,395 & 13,142 \\
\hline ICER & & & & $-52,568$ \\
\hline
\end{tabular}

a Other costs included were those of the prevention and treatment of febrile neutropenia and peripheral neuropathy
- exclusion of unrelated health system costs.

Additionally, we relied on these expected valueonly analyses for incremental cost-effectiveness ratios (ICERs). Similar to parameter uncertainty, many simulations resulted in negative QALYs and sometimes also negative costs, making the mean and median ICERs difficult to interpret.

We also performed a range of one-way sensitivity analyses, using the 2.5th and 97.5th percentile values of the input parameters to assess which ones contributed the most to uncertainty in the model QALYs and incremental cost outputs, but not the ICERs for the reason given above. 
Finally, we also performed net monetary benefit (NMB) analysis and produced cost-effectiveness acceptability curves using these NMB values rather than ICERs because of the greater validity of NMBs in this instance given the occurrence of negative ICERs. A cost-effectiveness threshold of \$US 34,240 per QALY was set for NMB analysis. This threshold equated to the China gross domestic product (GDP) per capita in 2015.

\section{Sensitivity analysis}

To perform probabilistic sensitivity analysis, we assigned distributions to the model parameters to represent the uncertainty associated with point estimates. We applied Monte Carlo simulation to generate 1000 runs and identified the 25th and 97.5th ranks as the corresponding 95\% confidence intervals (CIs).A beta-distribution was used to represent the uncertainty in utility,probability and proportions because these are binomial parameters that are constricted in the interval from zero to one. A gammadistribution was used for cost data.Based on the results of PSA, a cost-effectiveness acceptability curve was plotted to show the proportion of cost-effective simulations at different levels of willingness to pay per QALY gained.

Moreover, We also performed one-way sensitivity analyses. We varied the age at diagnosis and evaluated the impact of changes to assumptions regarding treatment decisions and the target population. We also extended the time period during which patients were at risk for recurrence, varied the discount rate, applied utility weights from other sources, applied wages of government workers to value the patient time, doubled the cost assigned for chemotherapy, and applied higher costs for distant recurrence.

\section{Results}

Base-case treatment analyses

Our model calculated the lifetime cumulative costs, quality adjusted life years.

(QALYs), incremental QALYs, incremental costs, incremental cost-effectiveness ratios(ICERs), and incidences of death for the five treatment strategies (Table 2).

The Markov model calculated that the four distribution proportions of the ACTH program were stabilization $(1.1 \%)$, remission $(38.2 \%)$, relapse $(58.2 \%)$ and death (2.6\%). Additionally, the four distribution proportions of the $\mathrm{TCH}$ program were stabilization $(0.2 \%)$, remission (46.3\%), relapse $(52.1 \%)$ and death $(1.4 \%)$. The 5 -y OS of $\mathrm{TCH}$ treatment was more than that of $\mathrm{ACTH}$ treatment (see Fig. 2).

Under our baseline assumptions, the model results showed that $\mathrm{TCH}$ was the dominant strategy: it was both less costly and offered more QALYs than the ACTH regimen. On average, TCH cost $\$ 13,142<\mathrm{ACTH}$. The effectiveness analysis showed that $\mathrm{TCH}$ was slightly preferred over the ACTH strategy when measured in QALYs (a difference of 0.25 QALYs). In the treatment of ACTH, PR of the breast cancer was achieved in 15 patients (60\%), stable disease was achieved in 3 patients (12\%), and seven patients $(28 \%)$ progressed. However, in the treatment of $\mathrm{TCH}, \mathrm{CR}$ of the breast cancer was achieved in 1 patient (6.25\%), PR of the breast cancer was achieved in 13 patients $(81.25 \%)$, stable disease was achieved in 1 patient (6.25\%), and 1 patient (6.25\%) progressed (See Table 2 ).

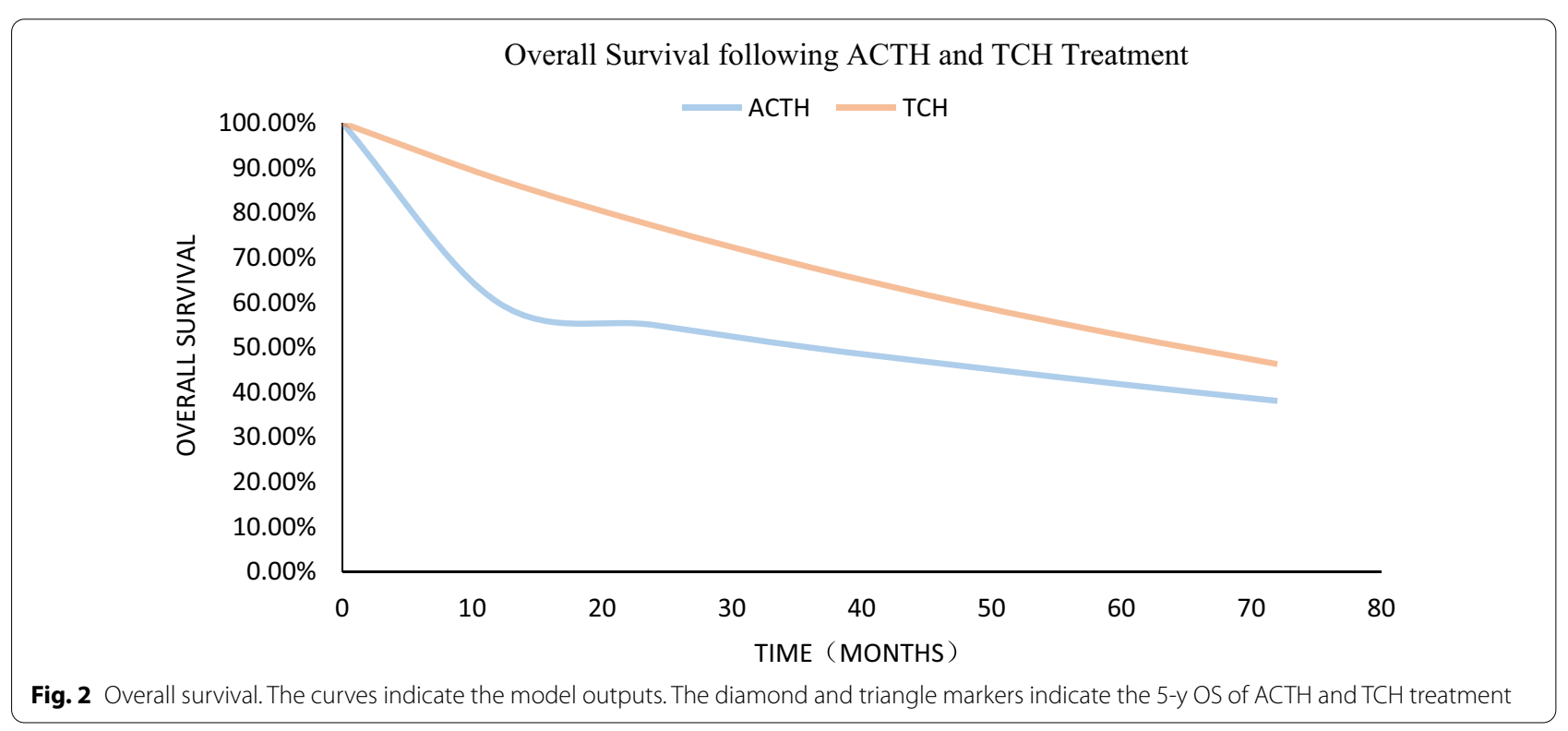


The QALYs gain was greater with $\mathrm{TCH}$ than with ACTH, with an estimated incremental QALY gain of 0.25 for ACTH versus TCH (Table 2). Additionally, the treatment of ACTH had greater costs. However, the extreme of the uncertainty intervals (UIs, i.e., the negative 2.5th percentiles) included QALY losses (Table 2). The costs were also higher with $\mathrm{ACTH}$ than with $\mathrm{TCH}$. We used expected value analysis, where the incremental costs and QALYs were the same or close to the averages from the Monte Carlo simulations.

The economic outcomes of alternative strategies are presented in Fig. 3. The AC-TH strategy is the dominant strategy because it is the most effective and the least expensive of the two alternative strategies. Applying incremental analysis principles, the ICER for ACTH versus TCH was \$US52,568 per QALY gained (See Table 2).

\section{General safety}

The most common AEs (any grade) during the neoadjuvant period were anemia, infection, hepatotoxicity, renal toxicity, cardiac toxicity and neutropenia (Table 3 ). The most common grade 3-4 AEs were infection, hepatotoxicity and neutropenia (Table 3 ), consistent with the higher proportion of patients who received optional prophylactic bone marrow support in ACTH. Neutropenia recorded as "neutrophil count decreased" was reported in 7 patients $(28 \%)$ in the ACTH group and 1 patient (6\%) in the $\mathrm{TCH}$ group. No grade $5 \mathrm{AEs}$ were reported. The most common serious AEs were hepatotoxicity and infection. Ten patients (40\%) in the ACTH group experienced grade $3-4$ hepatotoxicity, while four patients $(25 \%)$ in the $\mathrm{TCH}$ group experienced grade 3-4 hepatotoxicity. In our study, the incidence of symptomatic cardiotoxicity

Table 3 Adverse events (G1-2 and G3-4) for ACTH vs TCH

\begin{tabular}{llllll}
\hline & \multicolumn{2}{l}{$\begin{array}{l}\text { ACTH Adverse } \\
\text { events }\end{array}$} & & \multicolumn{2}{l}{ TCH Adverse events } \\
\cline { 2 - 3 } \cline { 5 - 6 } & G1-2 & G3-4 & & G1-2 & G3-4 \\
\hline Anemia & $5(20 \%)$ & $4(16 \%)$ & & $4(25 \%)$ & $7(44 \%)$ \\
Infection & $3(12 \%)$ & $18(72 \%)$ & 0 & $5(31.25 \%)$ \\
Neutropenia & $5(20 \%)$ & $7(28 \%)$ & $2(12.5 \%)$ & $1(6 \%)$ \\
Hepatotoxicity & $2(8 \%)$ & $10(40 \%)$ & & $1(6 \%)$ & $4(25 \%)$ \\
Renal toxicity & $2(8 \%)$ & 0 & & $2(12.5 \%)$ & 0 \\
Cardiac toxicity & & & & $3(18.75 \%)$ & 0 \\
$\quad$ Symptomatic dysfunction & $8(32 \%)$ & 0 & & 0 & 0 \\
Cardiac failure & 0 & 0 & & & \\
\hline
\end{tabular}

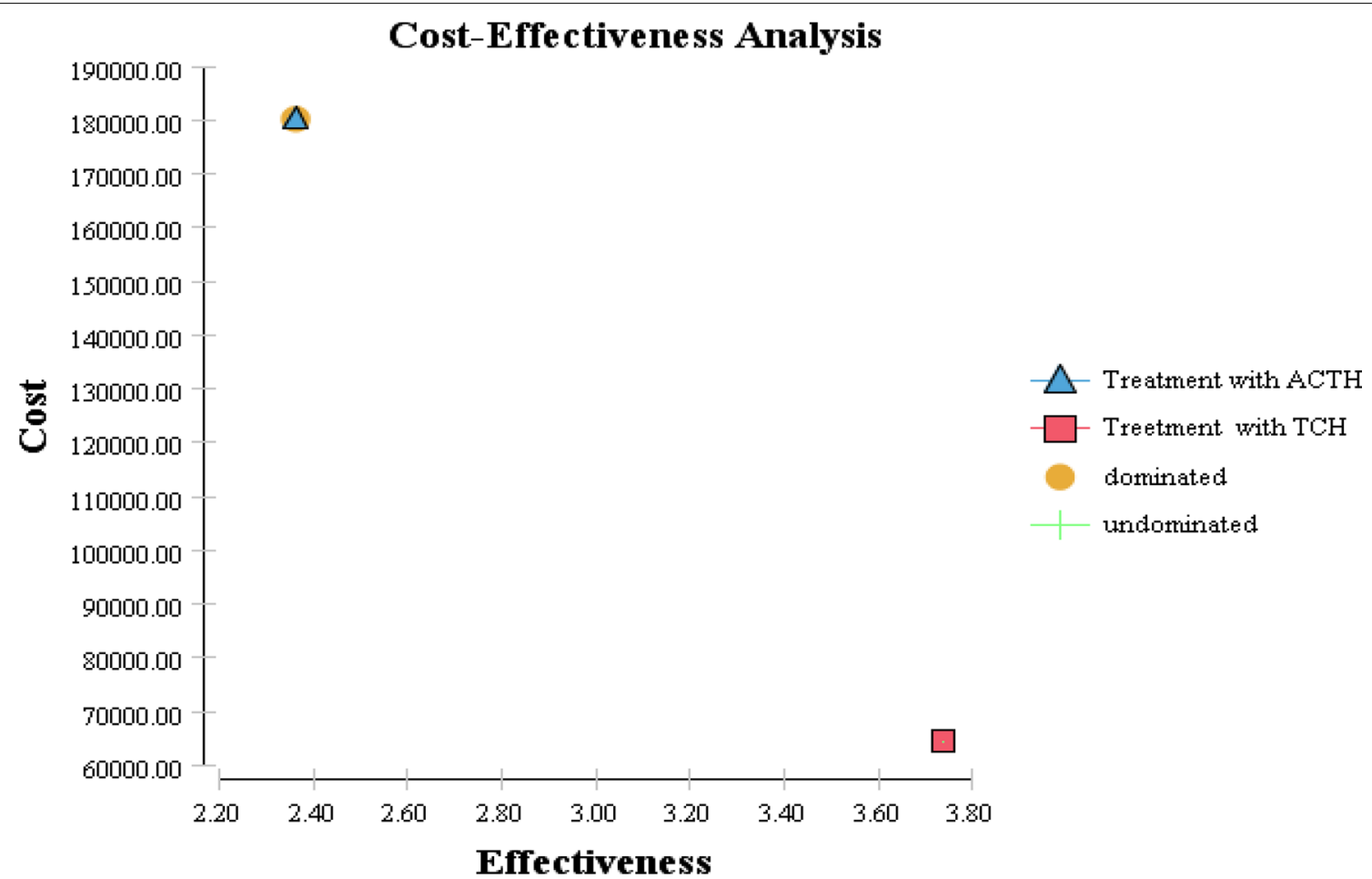

Fig 3. Results of cost-effectiveness analyses for the Breast cancer patients. The vertical axes represent the lifetime cumulative cost and the horizontal axes represent the quality-adjusted life years (QALYS) gained. The AC-TH strategy is the dominant strategy because it is the most effective and the least expensive of the two alternative strategies 
with ACTH treatment was $32 \%$, which was greater than that with $\mathrm{TCH}$ treatment.

\section{Sensitivity analyses}

\section{Single-factor sensitivity analysis}

Because of the method of calculating the transfer probability in this study was based on the literature data, the value of the corresponding transfer probability calculated from the maximum and minimum value in the literature data was used as the range of sensitivity analysis. Singlefactor sensitivity analysis was conducted using the sensitivity analysis method provided by TreeAge software. For both chemotherapy regimens, the transfer probability of remission to progression has the greatest impact on the model results. Using the tornado diagram function of TreeAge software, the tornado diagram of the transition probability is drawn, as shown in Fig. 4. The figure shows that the transition probability of the slow transfer to the progress state is an important variable influencing the conclusion of the model; thus, the variation of each transition probability within its sensitivity range would affect the change in the conclusion.

Because the drug prices in the study was from a hospital's data and the incidence of adverse reactions, adverse reactions costs were derived from real data, health utility data was from the literature.So all these parameters input in the Markov model conducted by single-factor sensitivity analysis using the sensitivity analysis method. To test the influence of these variables in the results of cost-effectiveness analysis. The tornado diagram of the transition probability is drawn, as shown in Fig. 4. The outcome of the one-way sensitivity analysis is reported as a "tornado diagram" (Fig. 4), and only the parameters that accounted for $99 \%$ of the cumulative risk related to the ICER are displayed.

The one-way sensitivity analyses revealed that some model parameters have a substantial impact on the results.The 5 most influential parameters are: the transition probability of $\mathrm{TCH}$ scheme from remission to remission (TCHrr), and the transition probability of $\mathrm{TCH}$ scheme from stabilization to remission (TCHsr), the transition probability (TPpd) of $\mathrm{TCH}$ scheme, the probability of transition from stable to stable of $\mathrm{TCH}$ scheme(TCHss), the transition probability of $\mathrm{TCH}$ scheme (TPsd).

Nevertheless, other parameters,including drug costs and other disease utilities, had little impact on the robustness of the model. When the threshold was set at $\$ 20,000$ per QALY, the above parameters have a

\section{Tornado Analysis (Net Benefits)}

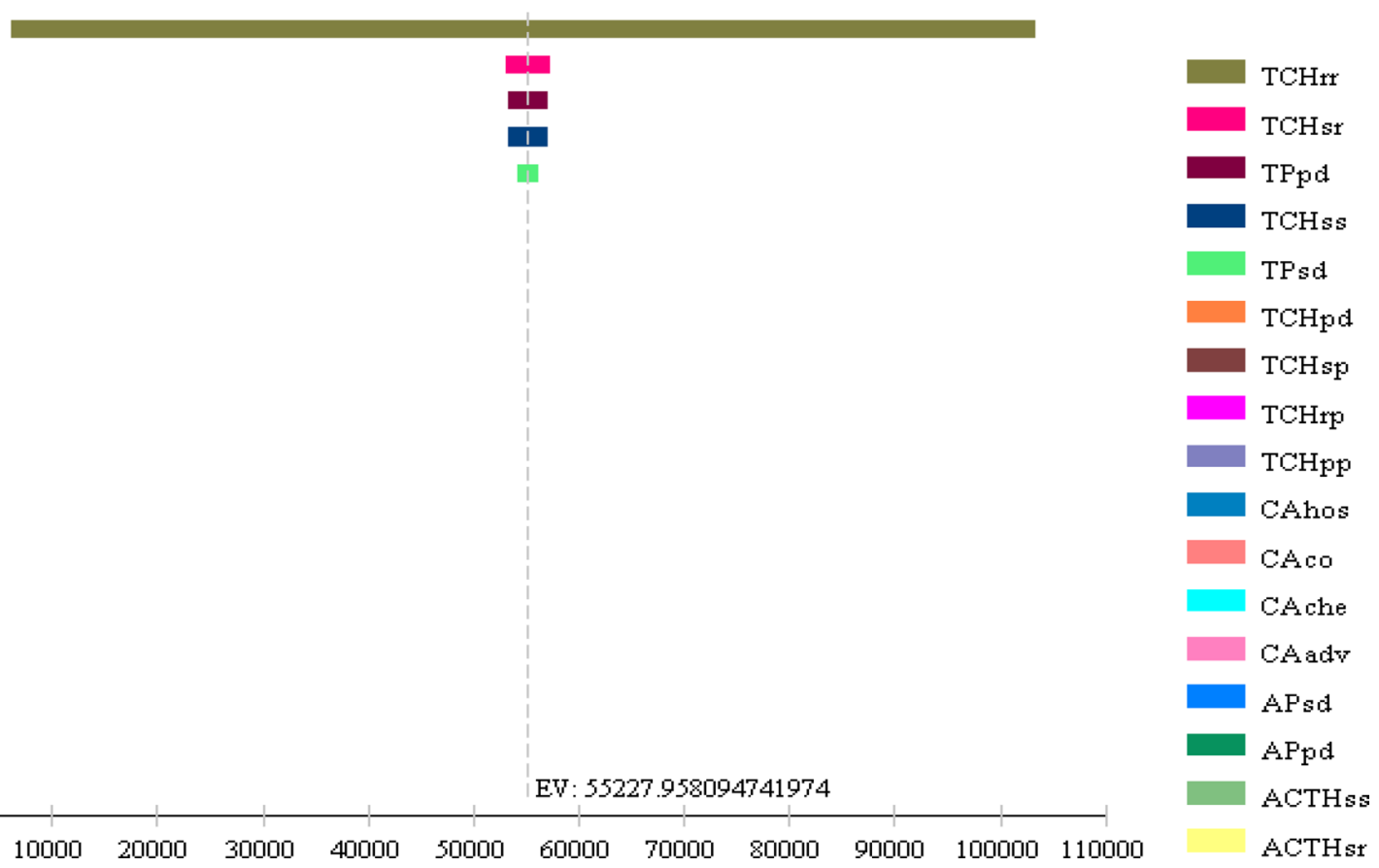

Fig. 4 Tornado diagram representing the cost per QALY gained in one-way sensitivity analysis for AC-TH strategy versus TCH strategy.The width of the bars represents the range of the results when the variables were changed. The width of the bars represents the range of results when the variables are changed.The vertical dotted line represents the base-case results 
certain influence on the ICER results in the sensitivity analysis range.

Because the health utility value of this study is only from a foreign literature, this parameter is unstable,but after sensitivity analysis of various parameters, it is concluded that the parameters that have a greater impact on the research results are mainly the $\mathrm{TCH}$ group probability of transition from remission to remission.

\section{Results of probability sensitivity analysis}

The probability sensitivity analysis uses the Monte Carl simulation method. Each simulation generates a Markov cohort of 10,000 people. The total number of Markov cohorts is 1000. After calculation,the two ICERs of neoadjuvant chemotherapy for breast cancer are compared. Furthermore,a probability sensitivity analysis can be performed. According to the Monte Carlo simulation results,the scatter plots of the $\mathrm{TCH}$ scheme and the
ACTH scheme are compared. The horizontal and vertical axes represent the incremental effect and incremental cost of the TCH scheme compared with the ACTH scheme. Each scatter in the figure represents the $\mathrm{TCH}$ scheme. The incremental cost-effectiveness ratio (ICER) of breast cancer with ACTH regimen is shown in the figure. Most of the scattered points in the figure are distributed in the first and fourth quadrants. The ICER ratio is generally negative,which shows that the TCH scheme has absolute advantages (see Fig. 5).

For a cohort of 1000 breast cancer patients, the costeffectiveness acceptable curve can be derived from the mutual comparison of the two schemes. It can be seen from Fig. 6 that within the WTP range, the probability of the ACTH scheme has a cost effect which is close to 0 , and the TCH scheme acceptance curve is maintained at a high level, which indicates that the $\mathrm{TCH}$ scheme has the highest acceptable probability and is the preferred solution.

\section{Incremental Cost-Effectiveness, Treetment with $\mathrm{TCH} v$. Treatment with ACTH}

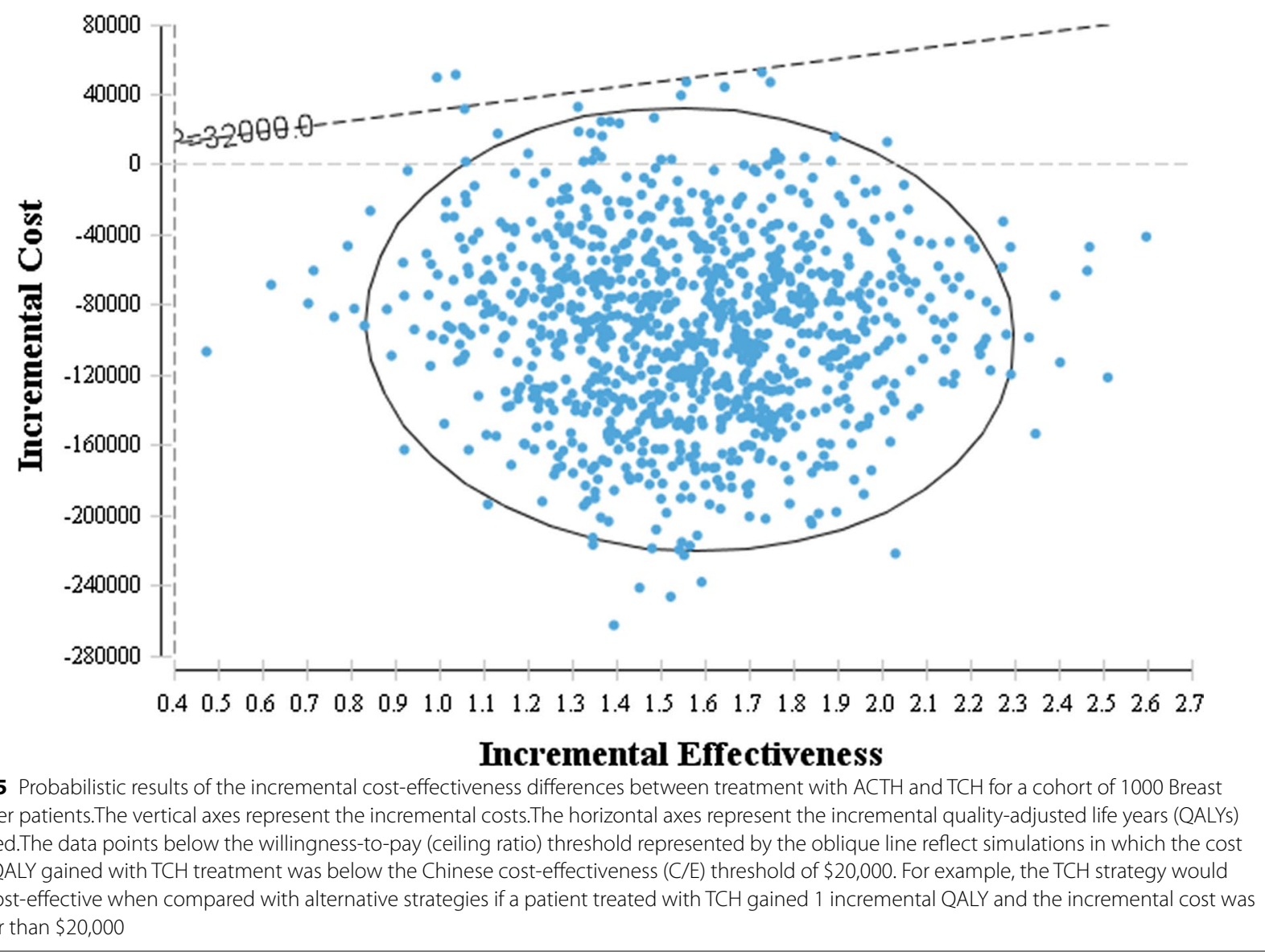




\section{Discussion}

The incidence of cardiotoxicity was increased when trastuzumab was used in combination with anthracyclines. The BCIRG-006 trial compared three chemotherapy regimens: doxorubicin, cyclophosphamide and docetaxel (AC-T), ACT plus trastuzumab (ACT-H), and docetaxel, carboplatin plus trastuzumab $(\mathrm{TCH})$ for the treatment of HER-2-positive breast cancer [8]. In this trial, both trastuzumab containing regimens (ACT-H and $\mathrm{TCH}$ ) were superior to ACT and similar to each other in terms of cancer efficacy. Importantly, $\mathrm{TCH}$ was associated with significantly less asymptomatic cardiotoxicity (>10\% decline in EF 9.4 vs. $18.6 \%$; $p<0.001$ ) and a lower incidence of symptomatic heart failure (0.4 vs. $2 \%$; $p<0.001)$ than ACTH [8]. In our study, the incidence of symptomatic cardiotoxicity of ACTH treatment was 32\%, which was more than that of $\mathrm{TCH}$ treatment. Additionally, in our study, the patients who had undergone ACTH treatment were also administered dexrazoxane, which is an effective iron chelator that reduces oxygen free radical production when administered with anthracyclines [25]. Swain and colleagues evaluated the data from three prospective studies to determine both the incidence of doxorubicin-related congestive heart failure CHF and the accumulated dose of doxorubicin at which $\mathrm{CHF}$ occurred [26]. The patients who received dexrazoxane had a significantly decreased incidence of cardiac events (defined as a decline from baseline $L V E F \geq 20 \%$, decline in LVEF $\geq 10 \%$ from baseline and $<$ lower limit of normal, or symptomatic CHF) compared with placebo [26]. Furthermore, they showed that dexrazoxane was cardioprotective even when it was given after patients had already received $300 \mathrm{mg} / \mathrm{m}^{2}$ of anthracyclines [27]. In our study, we found that with both $\mathrm{ACTH}$ and $\mathrm{TCH}$ treatment, none of the patients developed $\mathrm{CHF}$. The cause may be related to the drug conferring a protective effect on the patients' hearts. However, the FDA currently limits the use of dexrazoxane in women with metastatic breast cancer who need $>300 \mathrm{mg} / \mathrm{m}^{2}$ of anthracyclines. Due to concerns regarding decreased tumor response rates, increased myelosuppression and an increased incidence of the development of delayed hematologic malignancies, the routine use of dexrazoxane was not recommended in patients receiving anthraczycline therapy. We had consulted many references concerning dexrazoxane. A subsequent Cochrane meta-analysis had shown no difference in the oncologic response rates or incidence of secondary malignancies between patients receiving chemotherapy with or without dexrazoxane [28]. In this study, $28 \%$ of patients treated with the $\mathrm{AC}-\mathrm{TH}$ regimen had febrile

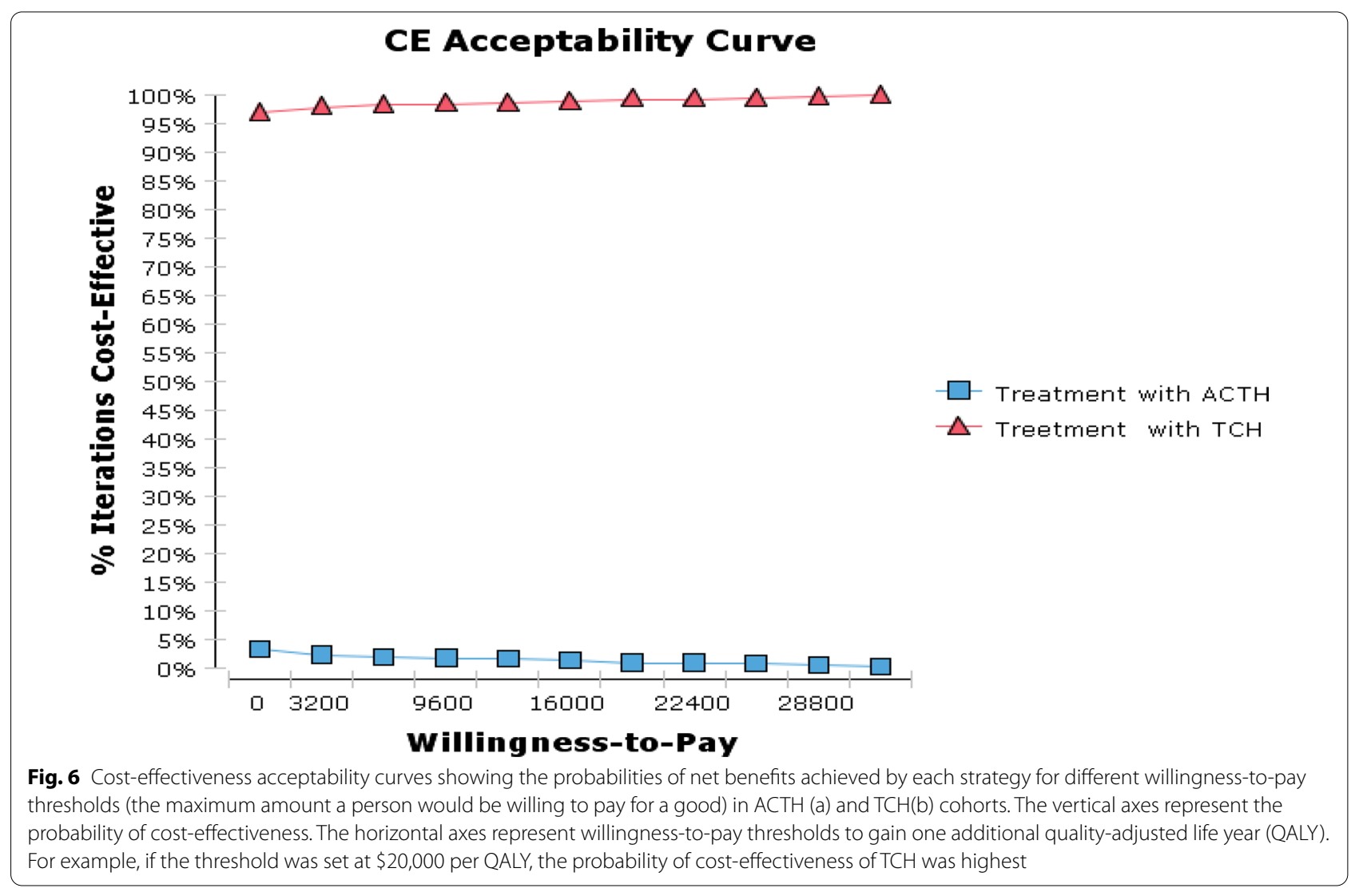


neutropenia, while the percent of febrile neutropenia was $6 \%$ in the $\mathrm{TCH}$ groups.

Re-evaluation of the cost effectiveness of the ACTH in the adjuvant treatment of breast cancer was timely given the emergence of $\mathrm{TCH}$ as a more effective option than the previous standard and significant changes in the relative costs of ACTH. For the first time, to our knowledge, the current economic analysis directly compared the cost effectiveness of ACTH and TCH in the adjuvant treatment of early breast cancer.

The results showed that $\mathrm{TCH}$ was likely to be a costeffective option compared with ACTH, with ICERs less than \$US 52,568 per QALY gained. While there was no set cost-effectiveness threshold in China, the ICERs were below the threshold based on the GDP per capita (approximately \$US 34,240 in 2015). In order to reduce the risk of death and suffering from disease, \$US52,568/ QALY should be acceptable to patients in our country. Thus, based on this model, TCH would be the preferred option if the health system was willing to pay the additional cost to benefit from the additional health gain. Indeed, our analysis showed that, at WTP thresholds above \$US 20,000 per QALY gained, TCH would have a greater probability of producing a more favorable NMB than ACTH. However, if the WTP threshold was less than this, ACTH would be preferred.

However, to our knowledge, no other cost-effectiveness analysis has directly compared different trastuzumab regimens, and the finding that $\mathrm{TCH}$ was cost effective compared with ACTH was not surprising. Jitawatanarat et al. [16] evaluated the tolerability and cardiac safety of docetaxel, cyclophosphamide, and trastuzumab (TCyH) for the treatment of early-stage human epidermal growth factor receptor-2 (HER2)-positive breast cancer and compared the standard trastuzumab-based chemotherapy regimens doxorubicin with cyclophosphamide followed by paclitaxel and trastuzumab $(\mathrm{ACTH})$ and docetaxel, carboplatin, and trastuzumab (TCaH). $\mathrm{TCyH}$ was well tolerated and should be investigated as an alternative adjuvant chemotherapy option for patients who are not candidates for standard trastuzumab-containing regimens when both were evaluated against non-taxane regimens [16].

Untch et al. [15] evaluated the efficacy and safety of epirubicin and cyclophosphamide followed by paclitaxel and trastuzumab as neoadjuvant treatment in patients with human epidermal growth factor receptor 2(HER2)-overexpressing breast cancer. Thirty-nine percent of the 217 enrolled patients achieved a pCR. The three-year disease-free survival (DFS) was $88 \%$ in patients with $\mathrm{pCR}$ compared with $73 \%$ in patients without $\mathrm{pCR}(P=0.01)$. The three-year overall survival (OS) was $96 \%$ in patients with pCR compared with
$86 \%$ in patients without $\mathrm{pCR}(P=0.025)$. Forty-eight recurrences were reported during a median follow up of 41 months corresponding to an estimated 3-year disease-free survival rate of $77.9 \%$.Twenty-three deaths were reported during post treatment observation, corresponding to an estimated 3-year OS rate of $89.4 \%$ [15]. Based on the results of the joint analysis of B-31 and NCCTG N9831, this benefit was considerable. At 4 years, the estimated absolute improvement in diseasefree survival is $18 \%$ (85-67\%; 95\% CI, $13-24 \%$ ), and for overall survival, the benefit was $4.8 \%$ (91.4-86.6\%; $95 \%$ CI, 0.6-9.0\%) [29].

Kolberg et al. [21] collected data from adjuvant trials and had shown that the combination of docetaxel, carboplatin and weekly trastuzumab $(\mathrm{TCH})$ was well tolerated and as effective as anthracycline-containing regimens. After a median follow up of 48.5 months, the disease-free survival (DFS) was $84.6 \%$, the distant disease-free survival (DDFS) was $87.2 \%$, and the overall survival (OS) was 91\%. Bayraktar et al. [19] evaluated the pathologic complete response (pCR) rates and relapse-free survival (RFS) and overall survival (OS) of patients receiving neoadjuvant systemic therapy (NST) with trastuzumab in combination with an anthracycline- or a non-anthracycline-based regimen. The pCR rates were 60.6 and $43.3 \%$ for patients who received PH-FECH $(\mathrm{n}=235)$ and TCH $(\mathrm{n}=65)$, respectively $(P=0.016)$. The three-year RFS rates were 93 and $71 \%$ $(P<0.001)$, and the 3 -years OS rates were $96 \%$ and $86 \%$ $(\mathrm{P}=0.008)$ for patients who received $\mathrm{PH}-\mathrm{FECH}$ and $\mathrm{TCH}$, respectively. Patients who received PH-FECH had a lower risk of recurrence (hazard ratio [HR]: 0.27; 95\% CI: $0.12-0.60 ; P=0.001)$ and death (HR: $0.37 ; 95 \%$ CI: $0.12-1.13 ; P=0.08)$ than those treated with TCH. Chen et al. [20] observed the efficacy of neoadjuvant trastuzumab combined with docetaxel and carboplatin $(\mathrm{TCH})$, and docetaxel, epirubicin and cyclophosphamide (TEC) chemotherapy in human epidermal growth factor receptor-2 (HER-2)-overexpressing breast cancer. The TCH group comprised 39 patients, and the TEC group comprised 25 patients. Neoadjuvant chemotherapy was continued for six cycles prior to comparison of the treatment efficacy.

\section{Study strengths and limitations}

(1) In this study, it was assumed that the transfer probability of the Markov model did not change during the study period. However, in the actual treatment process, the transfer probability between different states changed with time.

(2) The incidence of adverse reactions in different disease states of breast cancer was different, and the 
incidence of adverse reactions generally changed with time. The clinical truth data relied on by this study only provided the incidence of adverse reactions in the entire treatment group without providing the incidence of adverse reactions in different disease states.

(3) Due to the lack of research results on the health utility of different states of breast cancer in China, this study adopted the results of Ward et al.[13]

\section{Conclusion}

According to the traditional economic evaluation method, the cost of TCH chemotherapy is lower than that of the AC-TH regimen. Regarding the effect, the clinical yield of the TCH plan was higher than that of the $\mathrm{AC}-\mathrm{TH}$ plan, and the median survival time of $\mathrm{TCH}$ plan was longer than that of the AC-TH plan. Considering the cost and effect, the TCH plan is the best choice in both short-term and long-term economic evaluation. Incremental cost effect analysis showed that the TCH scheme is the preferred scheme with $\mathrm{AC}-\mathrm{TH}$ as the reference group.

\section{Acknowledgements}

This research was supported by the clinical pharmacist team of Zhejiang Cancer Hospital. We also acknowledge financial support by Shanghai Laishi Company

\section{Authors' contributions}

YW supervised the project in conception and execution, LYY, ZJJ and XQP performed measurements and analyzed data, LYY, LJ and LQY performed data validation, and XQP wrote the manuscript with input from LY, SCC, LYL and CLY. All authors read and approved the final manuscript.

\section{Funding}

This study has been funded by the Zhejiang Provincial Pharmaceutical Association Hospital Pharmacy Management Soft Science Research Funding Project of 2019 (Grant No. 2019ZYYG08). X.Q.P has been a master student in School of Respiratory pharmacology, Zhejiang University.

\section{Availability of data and materials Not applicable.}

\section{Ethics approval and consent to participate}

According to the ministry of health "measures on ethical review of biomedical research involving human beings (trial 2007)" 、WMA 《Declaration of Helsinki 》 and the ethical principles of CIOMS 《the international moral guide to human biological research》, Subject to review by the ethics committee, Agree to carry out this study according to the research scheme under review.I confirm that all methods were carried out in accordance with relevant guidelines and regulations. I confirm that all experimental protocols were approved by Ethics committee of hangzhou first people's hospital. I confirm that informed consent was obtained from all subjects.

\section{Consent for publication}

My manuscript is all about the two projects of breast cancer, I hope my article will help clinicians make decisions. I declare that there is no potential competitive interest in this article.l confirm that all authors have approved the manuscript for submission.I confirm that the content of the manuscript has not been published, or submitted for publication elsewhere.

\section{Competing interests}

The authors declare no competing interests.

\section{Author details}

${ }^{1}$ Department of Pharmacy, Hangzhou First People's Hospital, School of Medicine, Zhejiang University, Hangzhou 310006, China. ${ }^{2}$ Center for Healthcare Security Dig Data and Health Policy Studies, School of Public Health, Zhejiang University School of Medicine, 866 Yuhangtang Road, Hangzhou 310058, Zhejiang, China. ${ }^{3}$ Zhejiang Cancer Hospital, Hangzhou 310022, Zhejiang, China.

${ }^{4}$ Hangzhou Senile Hospital, Hangzhou 310022, Zhejiang, China.

Received: 20 June 2020 Accepted: 8 February 2021

Published online: 23 February 2021

\section{References}

1. Yang L, Wang J, Cheng JY, et al. Quality assurance target for communitybased breast cancer screening in China: a model simulation. BMC Cancer. 2018;18:261.

2. Slamon DJ, Leylandjones B, et al. Use of chemotherapy plus a monoclonal antibody against her 2 for metastatic breast cancer that overexpresses HER2. N Engl J Med. 2001;344:783-92.

3. Russell SD, Blackwell KL, Lawrence J, et al. Independent adjudication of symptomatic heart failure with the use of doxorubicin and cyclophosphamide followed by trastuzumab adjuvant therapy: a combined review of cardiac data from the National Surgical Adjuvant breast and Bowel Project B-31 and the North Central Cancer Treatment Group N9831 clinical trials. J Clin Oncol. 2010;28:3416-21.

4. Robert N, Leyland-Jones B, Asmar L, et al. Randomized phase III study of trastuzumab, paclitaxel, and carboplatin compared with trastuzumab and paclitaxel in women with HER-2-overexpressing metastatic breast cancer. J Clin Oncol. 2006;24:2786-92.

5. Russell SD, Blackwell KL, Lawrence J, et al. Independent adjudication of symptomatic heart failure with the use of doxorubicin and cyclophosphamide followed by trastuzumab adjuvant therapy: a combined review of cardiac data from the National surgical adjuvant breast and bowel project B-31 and the North central cancer treatment group N9831 clinical trials. J Clin Oncol. 2010;28:3416-21.

6. Procter M, Suter TM, de Azambuja E, et al. Longer-term assessment of trastuzumab-related cardiac adverse events in the herceptin adjuvant (HERA) trial. J Clin Oncol. 2010;28:3422-8.

7. Valero V, Forbes J, Pegram MD, et al. Multicenter phase III randomized trial comparing docetaxel and trastuzumab with docetaxel, carboplatin, and trastuzumab as first-line chemotherapy for patients with HER2-geneamplified metastatic breast cancer (BCIRG 007 study): two highly active therapeutic regimens. J Clin Oncol. 2011;29:149-56.

8. Slamon D, Eiermann W, Robert N, et al. Breast Cancer International Research Group, Adjuvant trastuzumab in HER2-positive breast cancer. N Engl J Med Econ. 2011;365:1273-83.

9. Donahue A, Mccune JS, Faucette S, et al. Measured versus estimated glomerular filtration rate in the Calvert equation: influence on carboplatin dosing. Cancer Chemother Pharmacol. 2001;47:373-9.

10. Diaby V, Ali AA, Adunlin G, et al. Parameterization of a disease progression simulation model for sequentially treated metastatic human epidermal growth factor receptor 2 positive breast cancer patients. Curr Med Res Opin. 2016:32:991-6.

11. Petrou PK, Talias MA. Cost-effectiveness of sorafenib compared to best supportive care in second line renal cell cancer from a payer perspective in Cyprus. Expert Rev Pharmacoecon Outcomes Res. 2014;14:131-8.

12. Purmonen T, Martikainen JA, Soini EJ, et al. Economic evaluation of sunitinib malate in second-line treatment of metastatic renal cell carcinoma in Finland. Clin Ther. 2008;30:382.

13. Ward S, Simpson E, Davis S, et al. Taxanes for the adjuvant treatment of early breast cancer: systematic review and economic evaluation. Health Technol Assess. 2007;11:1-144.

14. Romond EH, Perez EA, Bryant J, et al. Trastuzumab plus adjuvant chemotherapy for operable HER2-positive breast cancer. N Engl J Med. 2005;353:1673-84.

15. Untch M, Fasching PA, Konecny GE, et al. Pathologic complete response after neoadjuvant chemotherapy plus trastuzumab predicts favorable 
survival in human epidermal growth factor receptor 2-overexpressing breast cancer: results from the TECHNO trial of the AGO and GBG study groups. J Clin Oncol. 2011;29:3351-7.

16. Jitawatanarat $\mathrm{P}, \mathrm{O}^{\prime} \mathrm{C}$ onnor $\mathrm{TL}, \mathrm{Kossoff} E B$, et al. Safety and tolerability of docetaxel, cyclophosphamide, and trastuzumab compared to standard trastuzumab-based chemotherapy regimens for early-stage human epidermal growth factor receptor 2-positive breast cancer. J Breast Cancer. 2014;17:356-62

17. Ganz PA, Romond EH, Cecchini RS, et al. Long-term follow-up of cardiac function and quality of life for patients in NSABP protocol B-31/NRG oncology: a randomized trial comparing the safety and efficacy of doxorubicin and cyclophosphamide (AC) followed by paclitaxel with AC followed by paclitaxel and trastuzumab in patients with node-positive breast cancer with tumors overexpressing human epidermal growth factor receptor 2. J Clin Oncol. 2017;35:3942-8.

18. Dang C, Lin N, Moy B, et al. Dose-dense doxorubicin and cyclophosphamide followed by weekly paclitaxel with trastuzumab and lapatinib in HER2/neu-overexpressed/amplified breast cancer is not feasible because of excessive diarrhea. J Clin Oncol. 2010;28:2982-8.

19. Bayraktar S, Gonzalez-Angulo AM, Lei X, et al. Meric-Bernstam, Efficacy of neoadjuvant therapy with trastuzumab concurrent with anthracyclineand nonanthracycline-based regimens for HER2-positive breast cancer. Cancer. 2012;118:2385-93.

20. Chen W, He J, Song S, et al. Efficacy of TCH/TEC neoadjuvant chemotherapy for the treatment of HER-2-overexpressing breast cancer. Oncol Lett. 2015;9:1922-6.

21. Kolberg HC, Akpolat-Basci L, Stephanou M, et al. Neoadjuvant chemotherapy with docetaxel, carboplatin and weekly trastuzumab is active in HER2-positive early breast cancer: results after a median follow-up of over 4 years. Breast Care. 2016;11:323-7.

22. Echavarria I, Granja M, Bueno C, et al. Multicenter analysis of neoadjuvant docetaxel, carboplatin, and trastuzumab in HER2-positive breast cancer. Breast Cancer Res Treat. 2017:162:181-9.
23. Tiwari A, Gogia A, Deo S, et al. Retrospective study of efficacy and safety of neoadjuvant docetaxel, carboplatin, and trastuzumab in HER2-positive locally advanced and oligometastatic breast cancer: an Indian experience. Indian J Cancer. 2017;54:343-6.

24. Henderson IC, Berry DA, Demetri GD, et al. Improved outcomes from adding sequential paclitaxel but not from escalating doxorubicin dose in an adjuvant chemotherapy regimen for patients with node-positive primary breast cancer. J Clin Oncol. 2003;21:976.

25. Caron J, Nohria A. Cardiac toxicity from breast cancer treatment: can we avoid this? Curr Oncol Rep. 2018;20:61.

26. Swain SM, Whaley FS, Ewer MS. Congestive heart failure in patients treated with doxorubicin: a retrospective analysis of three trials. Cancer. 2003;97:2869-79.

27. Swain SM, Whaley FS, Gerber MC, et al. Delayed administration of dexrazoxane provides cardioprotection for patients with advanced breast cancer treated with doxorubicin-containing therapy. J Clin Oncol. 1997;15:1333-40

28. Shaikh F, Dupuis LL, Alexander S, et al. Cardioprotection and second malignant neoplasms associated with dexrazoxane in children receiving anthracycline chemotherapy: a systematic review and meta-analysis. J Natl Cancer Inst. 2016;108:357

29. Perez EA, Romond EH, Suman VJ, et al. Trastuzumab plus adjuvant chemotherapy for human epidermal growth factor receptor 2-positive breast cancer: planned joint analysis of overall survival from NSABP B-31 and NCCTG N9831. J Clin Oncol. 2014:32:3744-52.

\section{Publisher's Note}

Springer Nature remains neutral with regard to jurisdictional claims in published maps and institutional affiliations.
Ready to submit your research? Choose BMC and benefit from:

- fast, convenient online submission

- thorough peer review by experienced researchers in your field

- rapid publication on acceptance

- support for research data, including large and complex data types

- gold Open Access which fosters wider collaboration and increased citations

- maximum visibility for your research: over $100 \mathrm{M}$ website views per year

At BMC, research is always in progress.

Learn more biomedcentral.com/submissions 